\title{
Localized magnetic states in Rashba dots
}

\author{
Mircea Crisan, ${ }^{1}$ David Sánchez, ${ }^{2}$ Rosa López, ${ }^{2}$ Llorenç Serra, ${ }^{2,3}$ and Ioan Grosu ${ }^{1}$ \\ ${ }^{1}$ Department of Theoretical Physics, University of Cluj, 3400 Cluj, Romania \\ ${ }^{2}$ Departament de Física, Universitat de les Illes Balears, E-07122 Palma de Mallorca, Spain \\ ${ }^{3}$ Instituto de Física Interdisciplinar y Sistemas Complejos (CSIC-UIB), E-07122 Palma de Mallorca, Spain \\ (Received 7 November 2008; revised manuscript received 22 January 2009; published 20 March 2009)
}

\begin{abstract}
We study the formation of local moments in quantum dots arising in quasi-one-dimensional electron wires due to localized spin-orbit (Rashba) interaction. Using an Anderson-type model to describe the occurrence of the magnetic moments in these Rashba dots, we calculate the local magnetization within the mean-field approximation. We find that the magnetization becomes a nontrivial function of the Rashba coupling strength. We discuss both the equilibrium and nonequilibrium cases. Interestingly, we obtain a magnetic phase which is stable at large bias due to the Rashba interaction.
\end{abstract}

DOI: 10.1103/PhysRevB.79.125319

PACS number(s): 73.23.-b, 75.20.Hr, 71.70.Ej

\section{INTRODUCTION}

Spin-related phenomena have recently attracted much attention, as they are the key ingredient in the new field known as spintronics. ${ }^{1}$ Two-dimensional (2D) semiconductors are appropriate materials to be used in spintronics applications since they offer the possibility of an electric control of spins via a tunable spin-orbit (SO) interaction. An important contribution to $\mathrm{SO}$ effects in $2 \mathrm{D}$ electronic states of narrow gap semiconductors (e.g., InAs) is the Rashba interaction. ${ }^{2}$ This interaction is a generalization of the vacuum SO interaction from the Pauli equation, $H_{\mathrm{so}}=\left(e \hbar^{2} / 4 m^{2} c^{2}\right) \sigma \cdot[\nabla V(r) \times \mathbf{k}]$, which is small for nonrelativistic momenta $\hbar k \ll m c$, with $V(r)$ being the scalar potential. In semiconductors, the energy gap $E_{g}$ and the band splitting $\Delta$ are comparable in magnitude $\left(E_{g} \sim \Delta \sim 1 \mathrm{eV}\right)$, and as a consequence, the SO coupling is enhanced by a factor $m^{2} c^{2} / E_{g}$.

The Rashba interaction is a type of SO interaction that arises when a 2D electron gas forms at the interface of a heterostructure. To lowest order in momentum, the Rashba Hamiltonian reads

$$
H_{R}=\frac{1}{2 \hbar}\left(\left[\alpha, p_{y}\right]_{+} \sigma_{x}-\left[\alpha, p_{x}\right]_{+} \sigma_{y}\right),
$$

where $\alpha$ is the Rashba coupling proportional to the electric field producing the confinement. We take the confinement direction along $z$. In Eq. (1), $\mathbf{p}=\left(p_{x}, p_{y}\right)$ is the $2 \mathrm{D}$ momentum and $\sigma_{i}(i=x, y, z)$ are the Pauli matrices.

We note that available experimental data $^{3}$ on few-electron quantum dots have been discussed in terms of Rashba spinorbit coupling and exchange interaction. ${ }^{4}$ Using the spin density-functional theory it was showed ${ }^{5}$ that the competition of this coupling and the exchange interaction gives rise to the suppression of the Hund rule, and a dot with a closed configuration presents a paramagnetic behavior. We have to mention that these results have been obtained in the absence of the Coulomb interaction.

When the Rashba interaction is localized around a finite region of a quasi-one-dimensional ballistic wire [see Fig. 1(a)], Ref. 6 predicts the formation of quasi-bound-states which are coupled to the nonresonant background channel. Both the potential well and the intersubband coupling are produced by the Rashba interaction alone. Furthermore, the quasi-bound-states lead to enhanced backscattering, causing strong dips in the conductance curves of the wire as a function of the Fermi energy. ${ }^{7}$ Since both the level position and broadening can be tuned with the Rashba strength $\alpha$, these states are termed Rashba dots. ${ }^{6}$

Recently, López et al. ${ }^{8}$ formulated a microscopic theory for transport across Rashba dots including Coulomb interactions in the dot. An important aspect of this model is that different regimes can be achieved by tuning the parameters of the Rashba Hamiltonian, and this can be done by modulating external electric fields applied to nearby gates. The difference between the Anderson Hamiltonian ${ }^{9}$ and the Hamiltonian proposed in Ref. 8 is twofold. First, in the Anderson Hamiltonian the spin is conserved at low temperatures, leading to the Kondo effect, but in Ref. 8 the Rashba dot Hamiltonian contains a spin-flip interaction because the localized states couple to the continuum states with opposite spins. Second, due to the Rashba-induced precession term, the direct transmission channel presents a phase term similar to the Aharonov-Bohm case but the phase is now spin

a)

\begin{tabular}{c|c|c}
$\substack{\uparrow \\
\alpha \backslash \downarrow \downarrow \downarrow \\
\alpha \neq 0}$ & $\alpha=0$ \\
\hline
\end{tabular}

b)

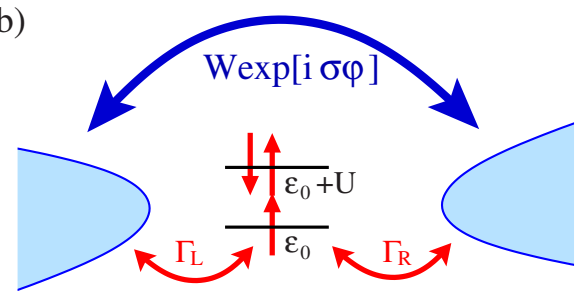

FIG. 1. (Color online) (a) The system under study consists of a quantum wire with a region of localized spin-orbit interaction of the Rashba type ( $\alpha$ is the Rashba coupling strength). Interactions are restricted to the Rashba dot. (b) Mapping of the upper system onto a quasilocalized level and a nonresonant background channel with spin-dependent couplings to external leads. 
dependent. ${ }^{10}$ Remarkably, despite these differences the system shows a persisting Kondo effect at low temperatures but with a novel gate dependence. ${ }^{8}$

In this paper, we address the magnetic properties of Rashba dots. We follow Anderson's model for magnetic impurities in a metallic host and determine whether it is energetically favorable for the dot to form a localized magnetic moment. We show below that the Coulomb interaction can develop magnetic moments in a Rashba dot for a critical value of the ratio $(U / \Gamma)_{\text {crit }}$, which depends on the parameters of the Rashba interaction. Our results might also be important for quantum dots doped with magnetic impurities. ${ }^{11,12}$ Magnetic ordering in dots can be induced by the Coulomb interaction, and the magnetization can be electrically controlled even for a fixed number of electrons. ${ }^{13,14}$

This paper is organized as follows. We present in Sec. II the model and calculate the Green's function using the equation-of-motion method. The magnetic moment is determined in Sec. III both for the equilibrium and the nonequilibrium cases. The main results are compared with exact numerical calculations in Sec. IV. The results are discussed in Sec. V, which also contains our conclusions.

\section{THEORETICAL MODEL}

We start with the model Hamiltonian

$$
H=H_{e}+H_{d}+H_{W}+H_{V},
$$

where

$$
\begin{gathered}
H_{e}=\sum_{\alpha, k, \sigma} \epsilon_{\alpha, k, \sigma} c_{\alpha, k, \sigma}^{\dagger} c_{\alpha, k, \sigma}, \\
H_{d}=\sum_{\sigma} \epsilon_{d} d_{\sigma}^{\dagger} d_{\sigma}+U n_{\sigma} n_{-\sigma}, \\
H_{W}=\sum_{k, \sigma}\left(W e^{i s_{\sigma} \varphi} c_{L, k, \sigma}^{\dagger} c_{R, k, \sigma}+\text { H.c. }\right), \\
H_{V}=\sum_{\alpha, k, \sigma}\left(V c_{\alpha, k, \sigma}^{\dagger} d_{-\sigma}+\text { H.c. }\right) .
\end{gathered}
$$

In this Hamiltonian we consider the spin quantization axis along the Rashba field (the $y$ direction for transport along $x$ ), $n_{\sigma}=d_{\sigma}^{\dagger} d_{\sigma}$ is the occupation number for electrons in the Rashba dot with spin $\sigma=\uparrow, \downarrow$, and $c_{\alpha, k, \sigma}^{\dagger}$ is the creation operator of continuum electrons with wave vector $k$ and spin $\sigma$ in the lead $\alpha=L, R$. The nonresonant channel is described with the term $H_{W}$ where the propagation phase acquired by a transmitted electron is spin dependent $\left(s_{\sigma}= \pm 1\right.$ if $\left.\sigma=\uparrow, \downarrow\right)$. Finally, localized and extended electronic states are coupled via the interaction $H_{V}$. A pictorial representation of $H$ is shown in Fig. 1(b).

The parameters of this Hamiltonian are $U=U(\alpha, l), V$ $=V(\alpha, l)$, and $W=W(\alpha, l)$, where $l$ is the length of the Rashba-induced square-well potential [we assume, for simplicity, that $\alpha(x)$ is constant if $0<x<l$ and zero otherwise], ${ }^{6}$ and $\varphi=k_{R} l$ with $k_{R}=m \alpha / \hbar^{2}$. Importantly, these parameters can be externally controlled with gates by changing $\alpha$ and $l$.
The form of $H$ is similar to the Hamiltonian describing the transport in a device formed by an Aharonov-Bohm interferometer with a quantum dot in one of its arms, ${ }^{15}$ but they differ in that the phases in the interaction term $W$ depend on the spin direction and that each hopping process through the dot is associated with a spin-flip event. In the conventional Anderson model, spin is conserved, and this leads to Kondo correlations.

In order to study the occurrence of magnetic moments in this model we will calculate the Green's function $G_{d \sigma}(\omega)$ $\equiv\left\langle\left\langle d_{\sigma} \mid d_{\sigma}^{\dagger}\right\rangle\right\rangle$, which obeys the equation

$$
\omega G_{d \sigma}(\omega)=1+\left\langle\left\langle\left[d_{\sigma}, H\right] \mid d_{\sigma}^{\dagger}\right\rangle\right\rangle .
$$

In the mean-field approximation, the spin-dependent energy of the $d$ electrons is $\epsilon_{d, \sigma}=\epsilon_{d}+U\left\langle n_{-\sigma}\right\rangle$. Using the general equation-of-motion method, we find

$$
\begin{aligned}
{\left[G_{d \sigma}(\omega)\right]^{-1}=} & \omega-\epsilon_{d \sigma}+\frac{1}{\Sigma} \sum_{\alpha k} \frac{2 V^{2}}{\omega-\epsilon_{k}} \\
& +\frac{4 W \cos \varphi}{\Sigma}\left(\sum_{k} \frac{V}{\omega-\epsilon_{k}}\right)^{2},
\end{aligned}
$$

where $\Sigma$ is given by the expression

$$
\Sigma=1-\left(\sum_{k} \frac{W}{\omega-\epsilon_{k}}\right)^{2} .
$$

If we perform the summations over $k$ in the relations above, the Green's function becomes

$$
G_{d \sigma}(\omega)=\left[\omega-\epsilon_{d \sigma}+i \Gamma+\Gamma \sqrt{x} \cos \varphi\right]^{-1},
$$

where $\Gamma=\Delta /(1+x), x=\pi^{2} W^{2} \nu^{2}$, and $\Delta=\pi V^{2} \nu$, with $\nu$ being the continuum density of states at the Fermi level $E_{F}$ (we take $\nu$ as a constant function of energy). We note that the tunneling broadening $\Delta$ becomes renormalized into $\Delta /(1$ $+x)$ due to the background channel when $W \neq 0$. Furthermore, the spin contribution due to the Rashba interaction is proportional to $\cos \varphi$ for both the spin orientations.

\section{MAGNETIC MOMENT}

\section{A. Equilibrium case}

The magnetization along the Rashba field direction is given by the difference between the occupancy expectation value for spin up and spin down,

$$
m=\left\langle n_{d \uparrow}\right\rangle-\left\langle n_{d \downarrow}\right\rangle .
$$

At zero temperature, the occupation reads

$$
\left\langle n_{d \sigma}\right\rangle=\int_{-\infty}^{E_{F}} \rho_{d}(\omega) d \omega,
$$

where $\rho_{d \sigma}(\omega)$ is the local density of states at the Rashba dot, defined in terms of the Green's function as $\rho_{d \sigma}(\omega)$ $=-\operatorname{Im} G_{d \sigma}(\omega) / \pi$.

Consider first the simple case $U=0$. Then, 


$$
\rho_{d \sigma}(\omega)=\frac{\Gamma}{\pi\left[\Gamma^{2}+\xi(\omega)^{2}\right]},
$$

where $\xi$ is given by

$$
\xi(\omega)=\omega-\epsilon_{d}+\Gamma \sqrt{x} \cos \varphi .
$$

Since $U=0$, the energy $\xi$ is spin independent. Inserting Eq. (10) in Eq. (9), we obtain

$$
\left\langle n_{d \sigma}\right\rangle=\frac{1}{2}-\frac{1}{\pi} \tan ^{-1} \frac{\epsilon_{d}-E_{F}-\Gamma \sqrt{x} \cos \varphi}{\Gamma} .
$$

Because $\left\langle n_{d \uparrow}\right\rangle=\left\langle n_{d \downarrow}\right\rangle$ even in the presence of spin-orbit interaction, we trivially have $m=0$. As expected, equilibrium magnetic states arise due to the presence of Coulomb interactions only.

Consider now the interacting case $U \neq 0$. We calculate the density of states using the spin dependence introduced by $\epsilon_{d \sigma}$ and we get

$$
\rho_{d \sigma}(\omega)=\frac{\Gamma}{\pi} \frac{1}{\xi_{-\sigma}(\omega)^{2}+\Gamma^{2}},
$$

where

$$
\xi_{-\sigma}(\omega)=\omega-\epsilon_{d}+\Gamma \sqrt{x} \cos \varphi-U\left\langle n_{-\sigma}\right\rangle .
$$

The occupation reads

$$
\left\langle n_{d \sigma}\right\rangle=\frac{1}{2}-\frac{1}{\pi} \tan ^{-1} \frac{\epsilon_{d}-E_{F}+U\left\langle n_{d,-\sigma}\right\rangle-\Gamma \sqrt{x} \cos \varphi}{\Gamma} .
$$

We analyze the formation of a magnetic state from the condition

$$
\frac{d\left\langle n_{d \sigma}\right\rangle}{d\left\langle n_{d,-\sigma}\right\rangle}=-U \rho_{d, \sigma}\left(E_{F}\right)
$$

As a consequence, the condition for the magnetic state that $d\left\langle n_{d}^{\sigma}\right\rangle / d\left\langle n_{d}^{-\sigma}\right\rangle<-1$ becomes

$$
U \rho_{d}^{\sigma}\left(E_{F}\right)>1 .
$$

This relation is similar to the Stoner condition for the occurrence of the magnetic state in the itinerant-electron systems, and the correlation effects appear only as an energy shift. A more accurate discussion, taking the energy dependence of $\Delta$ (via the density of states $\nu$ ) changes the magnetic region, which is known for a constant density of states. However, for our qualitative discussion we follow the wide-band approximation with a constant $\Delta$.

From Eqs. (8) and (15) we find a pair of self-consistent equations for the magnetization $m$ and the total electron density $n_{d}=\left\langle n_{d \uparrow}\right\rangle+\left\langle n_{d \downarrow}\right\rangle$,

$$
m=\frac{1}{\pi} \sum_{\sigma} s_{\sigma} \cot ^{-1} \frac{\frac{U}{2}\left(n_{d}-s_{\sigma} m\right)-\xi\left(E_{F}\right)}{\Gamma},
$$

$$
n_{d}=\frac{1}{\pi} \sum_{\sigma} \cot ^{-1} \frac{\frac{U}{2}\left(n_{d}-s_{\sigma} m\right)-\xi\left(E_{F}\right)}{\Gamma} .
$$

From these two equations we calculate the size of the interaction above which a local moment develops. On the critical boundary describing the transition into the magnetic state, we approximately have $m \approx 0$ and $\left\langle n_{d \uparrow}\right\rangle \approx\left\langle n_{d \downarrow}\right\rangle \approx n_{d}$. Thus, we find

$$
\left(\frac{U}{\Delta}\right)_{\text {crit }}=\frac{\pi\left(1+c^{2}\right)}{1+x},
$$

where $c=\cot \left(\pi n_{d} / 2\right)$. This condition provides a number of interesting predictions. First, for increasing $x$ the function $(U / \Delta)_{\text {crit }}$ decreases. Thus, the formation of magnetic moments is enhanced by the coupling to the continuum states, which is governed by the intensity of the Rashba interaction. Despite the fact that spin-orbit interactions are time-reversal symmetric and do not induce spontaneous magnetizations, indirectly the Rashba coupling makes it more favorable to generate magnetic solutions as compared to the case without Rashba interaction. If $x=0$ (or, equivalently, $W=0$ ) we recover the condition for the occurrence of the Anderson moments. ${ }^{9}$ Second, $(U / \Delta)_{\text {crit }}$ is a weakly function of the phase $\varphi$ since in Eq. (20) the dependence on $\varphi$ is only implicit through the total density $n_{d}$. Nevertheless, in the general case the condition given by Eq. (20) is far from being trivial since we recall that $U, \Delta$, and $n_{d}$ are complicated functions of the Rashba strength and the dot size. ${ }^{8}$

\section{B. Nonequilibrium case}

We now turn to the nonequilibrium case, where a finite dc bias $V$ is applied between the two electrodes. The formation of magnetic moments within the Anderson Hamiltonian out of equilibrium has been recently analyzed by Komnik and Gogolin, see Ref. 16. They found that the magnetic phase is stable at arbitrarily large voltages in the case of asymmetric couplings. Here, we assume symmetric couplings $\left(\Gamma_{L}=\Gamma_{R}\right)$ and show below that even in this case, the combination of Rashba interaction and finite bias leads to magnetic-moment formation.

At nonequilibrium, the spin-dependent occupations are given by the Keldysh (lesser) Green's function $G_{\sigma}^{<}\left(t, t^{\prime}\right)$ $=i\left\langle d_{\sigma}^{\dagger}(t) d_{\sigma}\left(t^{\prime}\right)\right\rangle$

$$
\left\langle n_{\sigma}\right\rangle=\int_{-\infty}^{\infty} \frac{d \omega}{2 \pi} \operatorname{Im} G_{\sigma}^{<}(\omega),
$$

where $G_{\sigma}^{<}(\omega)$ is the Fourier-transformed lesser Green's function. We find

$$
G_{\sigma}^{<}(\omega)=2 i \Gamma \frac{\sqrt{x} \sin \left(s_{\bar{\sigma}} \varphi\right)\left(f_{L}-f_{R}\right) /(1+x)+\left(f_{L}+f_{R}\right) / 2}{\xi(\omega)^{2}+\Gamma^{2}},
$$

where $f_{\alpha}=1 /\left[1+e^{\beta\left(\omega-\mu_{\alpha}\right)}\right]$ is the Fermi distribution function at the lead $\alpha=L, R$ with inverse temperature $\beta=1 / k_{B} T$. Interestingly enough, the occupation depends on a term propor- 
tional to $\sin (\bar{\sigma} \varphi)$ which has a different sign for opposite spins. This term appears only at nonequilibrium $\left(f_{L} \neq f_{R}\right)$. Therefore, we expect a spin polarization $(m \neq 0)$ for a noninteracting Rashba dot $(U=0)$ induced by the interplay effect of external bias and Rashba interaction. ${ }^{17}$

We take $\mu_{L}=E_{F}+e V / 2$ and $\mu_{R}=E_{F}-e V / 2$ for the electrochemical potentials in the left and right contacts. As a result, we obtain a closed expression for the magnetization,

$$
m=-\frac{\sqrt{T_{r}}}{\pi} \sin \varphi\left[\tan ^{-1} \frac{e V-2 \xi\left(E_{F}\right)}{2 \Gamma}+\tan ^{-1} \frac{e V+2 \xi\left(E_{F}\right)}{2 \Gamma}\right],
$$

where $T_{r}=4 x /(1+x)^{2}$ is the background channel transmission. We infer that the magnetization is negative for positive $V$, arising from the orientation of the effective Rashba field, which points along $-y .{ }^{18,19}$ The magnetization can be reversed if $\varphi$ changes sign (equivalently, the Rashba intensity $\alpha$ ). Obviously, the periodic dependence in Eq. (23) arises from the model but it is reasonable to assume a small $\alpha$. Therefore, $\varphi$ should not be very large. The periodic dependence on $\varphi$ is obtained in analogy with the Aharonov-Bohm effect and can be found in related spin-orbit systems (see, e.g., Ref. 17).

For $\varphi=\pi / 2$ the minimum magnetization reads

$$
m_{\min }=-2 \frac{\sqrt{T_{r}}}{\pi} \arctan \frac{e V}{2 \Gamma},
$$

which approaches -1 in the limits of $T_{r} \rightarrow 1$ and $e V \gg 2 \Gamma$. On the other hand, for gate voltages much larger than the applied bias, the magnetization approaches zero as

$$
m=-\frac{\sqrt{T_{r}}}{\pi} \frac{\Gamma e V}{\varepsilon_{d}^{2}+\Gamma^{2}} .
$$

Finally, we note that the magnetization becomes finite and independent of the gate voltage in the limit of infinite $V$, in which case we find the simple expression $m=-\sqrt{T_{r}}$.

In the interacting case, one must replace $\xi(\omega)$ with $\xi_{-\sigma}(\omega)$ in Eq. (22). Then, the expression becomes involved and the full phase diagram can be obtained only numerically. However, the model is tractable in special cases. In particular, we focus on level energies around the particle-hole symmetric point $\left(\varepsilon_{d}=-U / 2\right)$. We define the dimensionless parameters $p=-\varepsilon_{d} / U, y=U / \Gamma, z=e V / 2 \Gamma$, and $R=2(\Gamma / U) \sqrt{x} \cos \varphi$. Our goal is to characterize the critical line that separates the nonmagnetic and the magnetic phases. This is given by a curve $p_{c}$ versus $y_{c}$ in the $p-y$ plane for different values of $z$. Then, for small values of $p_{c}-1 / 2$ we find (see the Appendix),

$$
y_{c} \approx \frac{\pi}{2}\left(1+z^{2}\right)\left\{1+\left(1-3 z^{2}\right)\left[\frac{\pi}{2}\left(p_{c}-\frac{1}{2}\right)+\frac{R_{o}}{1+z^{2}}\right]^{2}\right\},
$$

where $R_{0}=\sqrt{x} \cos \varphi$. This result shows that the phase diagram presents a dip for $z>z^{*}$, where $z^{*}=1 / \sqrt{3}$ (as in Ref. 16), but the form of the phase diagram is modified by the Rashba parameter $R$. In fact, the dip position shifts away from the symmetric point due to the Rashba-induced level

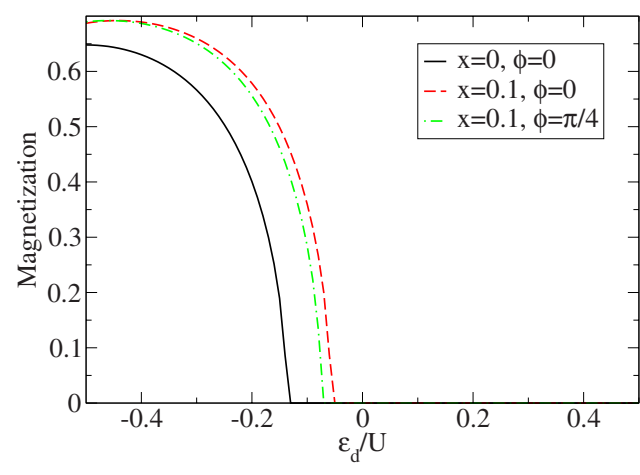

FIG. 2. (Color online) Equilibrium magnetization of the Rashba dot for $\Delta=0.2$ and the values of $x$ and $\varphi$ shown in the figure.

renormalization. The most important consequence is that whereas $m$ vanishes for $\varepsilon_{d}=-U / 2$ at large bias in the case without spin-orbit interactions, ${ }^{16}$ the magnetization remains finite in the Rashba case. We have numerically checked this prediction (see below).

\section{NUMERICAL RESULTS}

\section{A. Equilibrium case}

We now numerically solve Eqs. (8) and (15). For simplicity, we neglect the dependence of the system parameters on $\alpha$ and treat $U, \Delta$, and $\varepsilon_{d}$ as independent constants. In Fig. 2 we show $m$ as a function of $\varepsilon_{d}$ for $E_{F}=0$ and a fixed value of $\Delta$. In the absence of spin-orbit interaction $(x=0$ and $\varphi=0)$ the magnetization is zero for positive $\varepsilon_{d} / U$. When $\varepsilon_{d} / U$ decreases, there is a transition point into the magnetic state, whose magnetization becomes maximal at the particle-hole symmetric point $\left(\varepsilon_{d}=-U / 2\right)$. At this point, $m \approx 0.64$, in agreement with Ref. 9. We now change the value of $x$ and $\varphi$. These two parameters can be modified independently, tuning $\alpha$ and $l$. Then, for nonzero $x$ and $\varphi=0$ we find that the transition point shifts toward larger values of $\varepsilon_{d} / U$. This results from the self-energy shift $\Gamma \sqrt{x} \cos \varphi$ found in Eq. (7). Moreover, we observe an increase in the amplitude of $m$ as $x$ increases. This is a consequence of the Rashba-couplingenhanced magnetic-moment formation discussed above [Eq. (20)]. Furthermore, keeping $x$ constant and changing $\varphi$ we find that the magnetization curve changes only slightly, confirming our earlier prediction.

\section{B. Nonequilibrium case}

The nonequilibrium magnetization for the noninteracting case $(U=0)$ is shown in Fig. 3 for increasing values of the external bias $V$. The curves are symmetric around $\varepsilon_{d}=0$, which corresponds to the alignment between $\varepsilon_{d}$ and the Fermi energy. The magnetization is nonzero for all finite values of $V$, as discussed after Eq. (22). In the limit of large voltages, the curve becomes featureless according to Eq. (24).

The interacting case is shown in Fig. 4. All energies are given in units of $U=1$. For comparison, we also reproduce the curve corresponding to $U=0$ and $e V=0.1$. At the same voltage in the interacting case, we observe that the magnetization curve follows the noninteracting curve for large $\varepsilon_{d}$. 


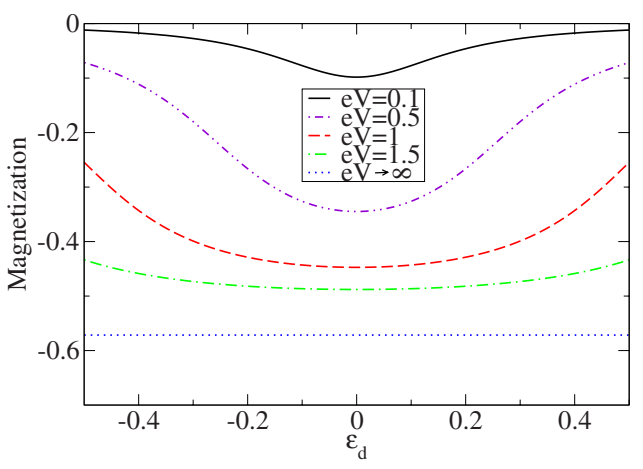

FIG. 3. (Color online) Nonequilibrium magnetization of the Rashba dot for $\Delta=0.2, x=0.1$, and $\varphi=\pi / 2$ as a function of the level position for different values of the bias voltage $V$ in the noninteracting case $(U=0)$.

This is reasonable since we are entering the empty orbital regime for which interactions are unimportant. In the opposite regime, i.e., for negative $\varepsilon_{d}$, strong correlations start to dominate and the interacting magnetization, although still finite, departs significantly from the noninteracting case. We obtain strong modifications in the magnetization curve for increasing voltages, favoring the development of magnetic moments due to the combined influence of interactions and spin-orbit coupling. For energies around the particle-hole symmetric point $\left(\varepsilon_{d} \approx-U / 2\right)$, we find that the magnetization is reduced as $V$ increases but unlike the case without spinorbit interactions, $m$ does not vanish in the limit of large bias. This is in excellent agreement with the prediction of Eq. (26).

\section{CONCLUSIONS}

We have studied the possibility of the occurrence of the magnetic models in a Rashba dot. The mean-field approximation has been used to calculate the magnetic moment and the critical value $U / \Delta$ for the occurrence of the local moments as a function of the parameters of the Rashba Hamiltonian. This condition, expressed by Eq. (20), is similar to the condition obtained for the Anderson model ${ }^{9}$ but contains also the parameter $x$ which is determined by the Rashba in-

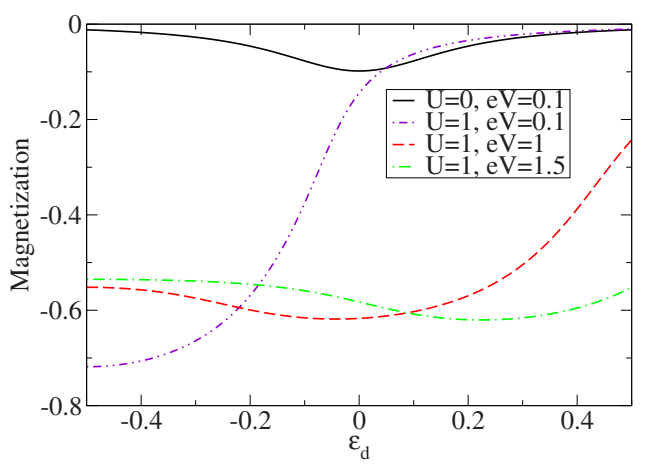

FIG. 4. (Color online) Nonequilibrium magnetization of the Rashba dot for $\Delta=0.2, x=0.1$, and $\varphi=\pi / 2$ as a function of the level position for different values of the bias voltage $V$ in the interacting case $(U=1)$. teraction. Therefore, our calculation suggests a driving of magnetic moments by external electric fields via the Rashba interaction. We have demonstrated that the value of the local magnetization $m$ at equilibrium depends on $x$, but it is worth noting that the curve $m\left(\epsilon_{d} / U\right)$ is not very sensitive to the change in the parameter $\varphi$. This result has been also shown in numerical simulations of the mean-field equations.

As in the standard Anderson calculation, ${ }^{9}$ our mean-field approach breaks the local symmetry, but in an exact solution accounting also for the effect of the spin fluctuations, we should recover the spin rotational invariance. Nevertheless, even if the magnetic states found above are an artefact of the model, the mean-field solution is interesting as such since it gives an indication of the region of the coupling constants of the Hamiltonian where the fluctuations give a relevant effect. Recently, magnetic-moment formation was proposed as a mechanism to explain the temperature dependence of the conductance for different gate voltages in quantum point contacts, ${ }^{20,21}$ where the scaling behavior of the conductance close to pinch off as a function of temperature was used as an argument for the Kondo effect occurrence. Hence, our results can be useful for these systems when spin-orbit interactions become relevant. We believe that our calculation can also be important for magnetic semiconductors. ${ }^{11,22}$

In the nonequilibrium regime, we have discussed the interplay between an external bias and the on-site interaction energy when the spin-orbit interaction is present. The phase diagram we obtain is different from the nonequilibrium case studied in Ref. 16, where the spin-orbit coupling was not considered. In Ref. 16, it is shown that the phase diagram presents a dip for $z>\frac{1}{\sqrt{3}}$. We have demonstrated that the spinorbit interaction yields in Eq. (26) a correction given by the last term proportional to $R_{0}$. In the case of symmetric model $\left(2 \epsilon_{d}+U=0\right)$ and large bias, the magnetization vanishes in the absence of spin-orbit interaction. ${ }^{16}$ In contrast, here we predict that the magnetization remains finite due to the Rashba interaction. Our numerical solution confirms this result, which can be particularly relevant for the experiments. It suggests that in materials with Rashba spin-orbit interaction the main contribution to the magnetization can be enhanced by applying a dc bias.

As possible extensions of our model, an interesting possibility is to take into account an energy-dependent density of states (specific for the semiconductors) such as the gapless density of states $\rho(\omega) \sim|\omega|^{r}$. This will give rise to an energydependent $\Delta(\omega)$, and the resulting behavior will likely differ from standard quantum dots. ${ }^{23}$ Future investigations could also deal with the effect of correlations which was neglected in the present calculations. Using the Hewson ${ }^{24}$ decoupling one might follow the method from Ref. 25 to calculate the effect of magnetic correlations in the $U \rightarrow \infty$ limit for systems with spin-orbit interaction. Finally, progress of experimental studies will be crucial for the directions in the development of this model.

\section{ACKNOWLEDGMENTS}

This work was supported by the Spanish Grant No. FIS2008-00781 from the "Ministerio de Ciencia e Innovación" and the "Ramón y Cajal" program. 


\section{APPENDIX}

We present here a derivation of Eq. (26). The mean occupations $n_{\uparrow}$ and $n_{\downarrow}$ have been calculated from the following relations:

$$
\begin{aligned}
2 \pi n_{\uparrow}= & -\sqrt{T_{r}} \sin \varphi\left[\tan ^{-1} \frac{e V+2 \xi_{\downarrow}\left(E_{F}\right)}{2 \Gamma}\right. \\
& \left.+\tan ^{-1} \frac{e V-2 \xi_{\downarrow}\left(E_{F}\right)}{2 \Gamma}\right] \\
& +\pi+\tan ^{-1} \frac{e V+2 \xi_{\downarrow}\left(E_{F}\right)}{2 \Gamma}-\tan ^{-1} \frac{e V-2 \xi_{\downarrow}\left(E_{F}\right)}{2 \Gamma}, \\
2 \pi n_{\downarrow}= & \sqrt{T_{r}} \sin \varphi\left[\tan ^{-1} \frac{e V+2 \xi_{\uparrow}\left(E_{F}\right)}{2 \Gamma}+\tan ^{-1} \frac{e V-\xi_{\uparrow}\left(E_{F}\right)}{2 \Gamma}\right] \\
& +\pi+\tan ^{-1} \frac{e V+2 \xi_{\uparrow}\left(E_{F}\right)}{2 \Gamma}-\tan ^{-1} \frac{e V-2 \xi_{\uparrow}\left(E_{F}\right)}{2 \Gamma} .
\end{aligned}
$$

Using these results we obtain the magnetization $m$,

$$
\begin{aligned}
m= & -\frac{1}{2 \pi} \sqrt{T_{r}} \sin \varphi\left[\tan ^{-1} \frac{e V+2 \xi_{\downarrow}\left(E_{F}\right)}{2 \Gamma}+\tan ^{-1} \frac{e V-2 \xi_{\downarrow}\left(E_{F}\right)}{2 \Gamma}\right. \\
& \left.+\tan ^{-1} \frac{e V+2 \xi_{\uparrow}\left(E_{F}\right)}{2 \Gamma}+\tan ^{-1} \frac{e V-2 \xi_{\uparrow}\left(E_{F}\right)}{2 \Gamma}\right] \\
& +\frac{1}{2 \pi}\left[\tan ^{-1} \frac{e V+2 \xi_{\downarrow}\left(E_{F}\right)}{2 \Gamma}-\tan ^{-1} \frac{e V-2 \xi_{\downarrow}\left(E_{F}\right)}{2 \Gamma}\right. \\
& \left.-\tan ^{-1} \frac{e V+2 V \xi_{\uparrow}\left(E_{F}\right)}{2 \Gamma}+\tan ^{-1} \frac{e V-2 \xi_{\uparrow}\left(E_{F}\right)}{2 \Gamma}\right] .
\end{aligned}
$$

From this expression we can see that for $U=0$ we have $\xi_{\uparrow}\left(E_{F}\right)=\xi_{\downarrow}\left(E_{F}\right)=\xi\left(E_{F}\right)$ and the magnetization $m$ has the value given in Eq. (23) with $T_{r}=4 x /(1+x)^{2}$.

In the same way we calculate the total occupation $n=n_{\uparrow}$ $+n_{\downarrow}$ as

$$
\begin{aligned}
n= & -\frac{1}{2 \pi} \sqrt{T_{r}} \sin \varphi\left[\tan ^{-1} \frac{e V+2 \xi_{\downarrow}\left(E_{F}\right)}{2 \Gamma}+\tan ^{-1} \frac{e V-2 \xi_{\downarrow}\left(E_{F}\right)}{2 \Gamma}\right. \\
& \left.-\tan ^{-1} \frac{e V+2 \xi_{\uparrow}\left(E_{F}\right)}{2 \Gamma}-\tan ^{-1} \frac{e V-2 \xi_{\uparrow}\left(E_{F}\right)}{2 \Gamma}\right] \\
& +\frac{1}{2 \pi}\left[\tan ^{-1} \frac{e V+2 \xi_{\downarrow}\left(E_{F}\right)}{2 \Gamma}-\tan ^{-1} \frac{e V-2 \xi_{\downarrow}\left(E_{F}\right)}{2 \Gamma}\right.
\end{aligned}
$$

$$
\left.+\tan ^{-1} \frac{e V+2 \xi_{\uparrow}(F)}{2 \Gamma}-\tan ^{-1} \frac{e V-2 \xi_{\uparrow}\left(E_{F}\right)}{2 \Gamma}+2 \pi\right],
$$

which for $U=0$ becomes

$$
n=1+\frac{1}{\pi} \tan ^{-1} \frac{8 \Gamma \xi}{4 \Gamma^{2}-4 \xi^{2}+(e V)^{2}}
$$

for $\left[(e V)^{2}-4 \xi^{2}\right] / 4 \Gamma^{2}>-1$. In the limit $V \rightarrow 0$ this equation gives, at $E_{F}=0$ and $\varphi=\pi / 2$,

$$
n=1-\frac{1}{\pi} \tan ^{-1} \frac{2 \Gamma \varepsilon_{d}}{\Gamma^{2}-\varepsilon_{d}^{2}} .
$$

From these relations we expect that the magnetic $\left(n_{\sigma} \neq n_{-\sigma}\right)$ and nonmagnetic solutions $\left(n_{-\sigma}=n_{\sigma}\right)$ exist for small $V$. In the following we will analyze the phase diagram taking into consideration the extra parameter $V$ and the Rashba interaction. We introduce ${ }^{9,16}$ the parameter $n_{c}$, which runs from 0 to 1 , and the dimensionless parameters $p=-\varepsilon_{d} / U, y=U / \Gamma, z$ $=e V / 2 \Gamma$, and $R=2(\Gamma / U) \sqrt{x} \cos \varphi$. Thus,

$$
\begin{aligned}
n_{c}= & 1+\frac{1}{\pi} \tan ^{-1}\left[z+y_{c}\left(p_{c}+R / 2-n+n_{c}\right)\right] \\
& -\frac{1}{\pi} \tan ^{-1}\left[z-y_{c}\left(p_{c}+R / 2-n+n_{c}\right)\right] .
\end{aligned}
$$

Deriving this expression with regard to $n_{c}$ we arrive at

$$
\begin{aligned}
\frac{\pi}{y_{c}}= & \frac{1}{1+\left[z+y_{c}\left(p_{c}+R / 2-n+n_{c}\right)\right]^{2}} \\
& +\frac{1}{1+\left[z-y_{c}\left(p_{c}+R / 2-n+n_{c}\right)\right]^{2}} .
\end{aligned}
$$

For $p_{c}=1 / 2$ we can fix $n_{c}=1 / 2$ and $n=1$,

$$
\frac{\pi}{y_{c}}=\frac{1}{1+\left(z+R_{0}\right)^{2}}+\frac{1}{1+\left(z-R_{0}\right)^{2}},
$$

where $R_{0}=\sqrt{x} \cos \varphi$. We now write down the equation which contains the small parameter $p_{c}-1 / 2$,

$$
\begin{aligned}
\frac{\pi}{y_{c}}= & \frac{1}{1+\left[y_{c}\left(p_{c}-1 / 2\right)+z+R_{0}\right]^{2}} \\
& +\frac{1}{1+\left[y_{c}\left(p_{c}-1 / 2\right)-z+R_{0}\right]^{2}} .
\end{aligned}
$$

Using Eq. (A9) we can solve Eq. (A10) iteratively, yielding Eq. (26).
${ }^{1}$ For a recent review, see J. Fabian, A. Matos-Abiague, C. Ertler, P. Stano, and I. Zutic, Acta Phys. Slov. 57, 565 (2007).

${ }^{2}$ E. I. Rashba, Fiz. Tverd. Tela (Leningrad) 2, 1224 (1960) [Sov. Phys. Solid State 2, 1109 (1960)].

${ }^{3}$ S. Tarucha, D. G. Austing, T. Honda, R. J. van der Hage, and L. P. Kouwenhoven, Phys. Rev. Lett. 77, 3613 (1996).
${ }^{4}$ L. P. Kouwenhoven, D. G. Austing, and S. Tarucha, Rep. Prog. Phys. 64, 701 (2001).

${ }^{5}$ M. Governale, Phys. Rev. Lett. 89, 206802 (2002).

${ }^{6}$ D. Sánchez and Ll. Serra, Phys. Rev. B 74, 153313 (2006).

${ }^{7}$ L. Zhang, P. Brusheim, and H. Q. Xu, Phys. Rev. B 72, 045347 (2005). 
${ }^{8}$ R. López, D. Sánchez, and Ll. Serra, Phys. Rev. B 76, 035307 (2007).

${ }^{9}$ P. W. Anderson, Phys. Rev. 124, 41 (1961).

${ }^{10}$ Y. Aharonov and A. Casher, Phys. Rev. Lett. 53, 319 (1984).

${ }^{11}$ Y. Léger, L. Besombes, J. Fernández-Rossier, L. Maingault, and H. Mariette, Phys. Rev. Lett. 97, 107401 (2006).

${ }^{12}$ J. Fernández-Rossier and R. Aguado, Phys. Rev. Lett. 98, 106805 (2007).

${ }^{13}$ I. Zutic, J. Fabian, and S. Das Sarma, Rev. Mod. Phys. 76, 323 (2004).

${ }^{14}$ R. M. Abolfath, P. Hawrylak, and I. Zutic, Phys. Rev. Lett. 98, 207203 (2007).

${ }^{15}$ B. R. Bułka and P. Stefański, Phys. Rev. Lett. 86, 5128 (2001); W. Hofstetter, J. König, and H. Schoeller, ibid. 87, 156803 (2001).

${ }^{16}$ A. Komnik and A. O. Gogolin, Phys. Rev. B 69, 153102 (2004).

${ }^{17}$ Similar bias-induced spin accumulations have been reported for quantum dots with Rashba interaction inserted in a mesoscopic interferometer by Q. F. Sun and X. C. Xie, Phys. Rev. B 73, 235301 (2006).

${ }^{18}$ Ll. Serra, D. Sánchez, and R. López, Physica E 40, 1479 (2008).

${ }^{19}$ P. Brusheim and H. Q. Xu, Phys. Rev. B 74, 205307 (2006).

${ }^{20}$ Y. Meir, K. Hirose, and N. S. Wingreen, Phys. Rev. Lett. 89, 196802 (2002).

${ }^{21}$ P. S. Cornaglia, C. A. Balseiro, and M. Avignon, Europhys. Lett. 67, 634 (2004); Phys. Rev. B 71, 024432 (2005).

${ }^{22}$ M. Ichimura, K. Tanikawa, S. Takahasi, G. Baskaran, and S. Maekawa, in Proceedings of ISQM-Tokyo '05, edited by S. Ishioka and K. Fujikawa (World Scientific, Singapore, 2006), pp. 183-186; arXiv: cond-mat/0701736.

${ }^{23}$ J. Hopkinson, K. Le Hur, and É. Dupont, Eur. Phys. J. B 48, 429 (2005); Physica B 359-361, 1454 (2005).

${ }^{24}$ A. C. Hewson, Phys. Rev. 144, 420 (1966).

${ }^{25}$ P. Stefański, A. Tagliacozzo, and B. R. Bułka, Solid State Commun. 135, 314 (2005). 